\title{
Human-interactive annealing for turning threat to opportunity in technology development
}

\author{
Yoshiharu Maeno*, Kiichi Ito**, Kenichi Horie***, and Yukio Ohsawa*** \\ * University of Tsukuba, Bunkyo-ku, Tokyo 112-0012 \\ ** Keio University, Fujisawa, Kanagawa 252-8520 \\ *** University of Tokyo, Bunkyo-ku, Tokyo 113-8563 \\ maeno.yoshiharu@nifty.com
}

\begin{abstract}
Human-interactive annealing is a new method for understanding invisible but relevant events, and inventing hypothetical scenarios. The human-interactive annealing is elaborated for texts. It is applied to patents for acquiring opportunity in technology development. An illustrative experiment on discovering opportunity by analyzing US patent texts on knowledge acquisition is demonstrated. A few sample hypothetical scenarios on emerging technological elements are obtained.
\end{abstract}

\section{Introduction}

In contrast to recent information flood, it is believed that invisible but relevant events are major origins of threat and opportunity in many social and business problem domains [1]. There are various kinds of invisible events which play an important role in the dynamics of visible events. Such invisible events are named "dark events" after dark matter in cosmology in physics. The invisibility problem is beyond the scope of conventional methods in chance discovery [2].

We developed a new method; "human-interactive annealing" [3], for understanding dark events, revealing a latent structure, and inventing hypothetical scenarios. The annealing is a combination of complementary elements; "human's interpretation", and "crystallization algorithm". The algorithm [4] is developed to locate, and to visualize dark events. Experiment on discovering an invisible leader under an on-line communication circumstance was demonstrated in [5].

In technology development [6], strategies on intellectual properties are critical to earning, costing, and even survival of companies. Decision-making on developing, buying, or licensing technologies is central to the strategies. There are a number of invisible technological elements, which are the major origin of "threat". A start-up company may try to develop an emerging technology. A company in different business domains may introduce a new technology and challenge to access the market. A competitor company may possess undisclosed surpassing technology. A sub-marine patent had been a great threat. If a company, however, has an ability to infer and invent a scenario on such invisible technological elements, the company could turn "threat" to "opportunity" to acquire the technological elements extensively, and exclusively.

In this paper, we elaborate the human-interactive annealing for analyzing texts. It is applied to patents for acquiring opportunity in technology development. An illustrative experiment on discovering opportunity by analyzing US patent texts on knowledge acquisition is demonstrated. A few sample scenarios regarding emerging technological elements are obtained.

\section{Human-interactive annealing}

The human-interactive annealing process aims at discovery from difference. The difference is between "internal construct of the mind" (human's work) and "external artifact supporting decision making" (computer's work) [7]. The human's work includes human's interpretation from individual persons. The computer's work includes crystallization algorithm to analyze observation, and to locate dark events. The dark events are invisible but relevant events, whose sign is inferred by the difference. Through the iteration of interaction between human's work and computer's work, we acquire understanding, and remove misunderstanding.

To acquire understanding means to move toward higher levels of understanding (Level 1: pre-structural, Level 2: uni-structural, Level 3: multi-structural, Level 4: relational, and Level 5: extended abstract [8]). The interaction between persons is useful to incorporate expert knowledge [9], and to deepen understanding. The overall process is illustrated in Fig.1.

A scenario map is used to connect crystallization algorithm to human's interpretation. The scenario map represents dark events as well as event clusters. The dark events are made visible, owing to the crystallization algorithm. We use peak temperature, as a control parameter representing the degree of understanding, to connect human's interpretation to crystallization algorithm. 


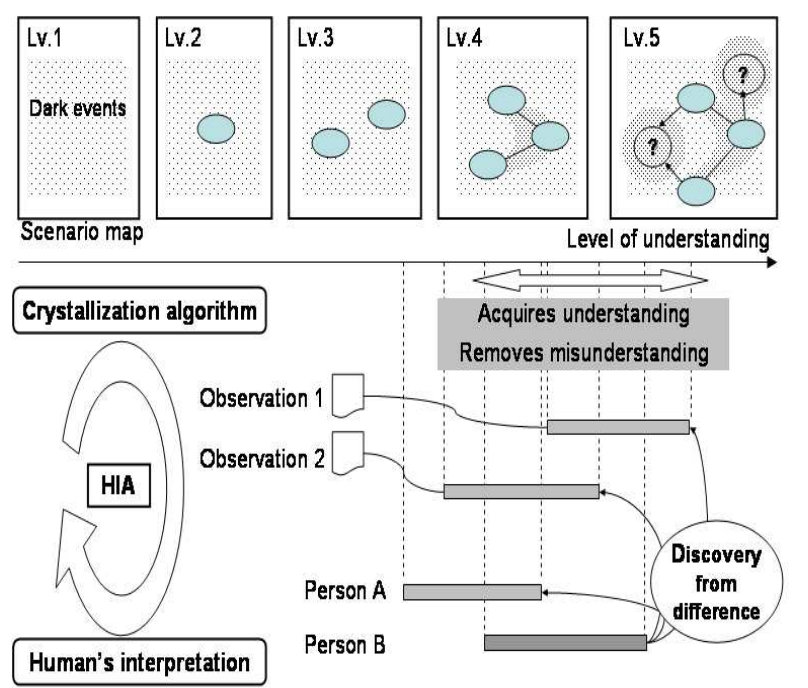

Fig.1 Overall process of the human-interactive
annealing.

The peak temperature is converted to the number of clusters and links on the scenario map. When the degree of understanding is recognized to be high, the peak temperature shall be set higher. When the degree of understanding is low, the peak temperature shall be set lower.

While annealing is executed with the modified peak temperature, heating step and cooling step occurs. In the heating step, up to the specified peak temperature, the number of clusters and edges between visible events decrease. Weak associations are destroyed. The crystallized dark events disappear. Then, a cooling step comes after the heating step, where event structures are solidified as temperature goes down. The number of crystallized dark events between event clusters increases on a scenario map. The clusters are connected to each other to form a single large structure.

The detailed procedure of the human-interactive annealing is shown in Fig. 2. At the beginning of the crystallization algorithm, the input text is morphologically analyzed. A sentence is converted into a basket; $b_{i}=\left\{e_{i}\right\}$. In "Execute event clustering", the events $e_{i}$ are classified into word groups. A word group is a cluster representing an idea. The idea should be interpreted mainly by identifying subjects, predicates, objects, or modifiers. Here, we employ k-medoid clustering algorithm for computational simplicity [10]. It is similar to an EM algorithm. A medoid event $e_{\text {medi }(i)}$ is a center of a cluster. It is repeatedly updated so that closeness from events within a cluster to the modoid can be maximal. Based on Jaccard coefficient by equation (1), the closeness $C l_{j}$ is evaluated by equation (2). Event partitioning into clusters is updated as well.

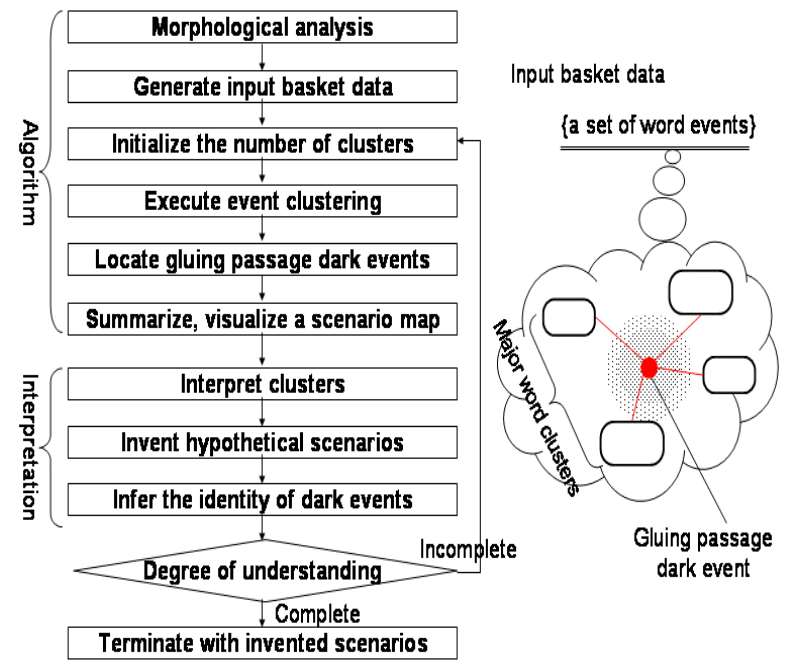

Fig.2 Procedure for crystallization algorithm and human's interpretation.

$$
\begin{aligned}
& \operatorname{Ja}\left(e_{i}, e_{j}\right) \equiv \frac{\operatorname{Freq}\left(e_{i} \cap e_{j}\right)}{\operatorname{Freq}\left(e_{i} \cup e_{j}\right)} \\
& C l_{j} \equiv \sum_{e_{j} \neq e_{\text {med }(i)}, e_{j} \in c_{j}} \mathrm{Ja}\left(e_{\text {med }(j)}, e_{j}\right),
\end{aligned}
$$

In "Locate gluing passage dark events", a dummy event $D E_{i}$ is inserted into each basket: $b_{i}=\left\{e_{i}, D E_{i}\right\}$. The index $i$ can be used to identify the basket where the corresponding dummy event was inserted. The dummy event represents dark events which potentially participate in the basket. Likelihood of a dummy event being a dark event $\mathrm{Co}_{i}$ is measured by Jaccard coefficient with the dummy event and event clusters, according to equation (3). According to the value of equation (3), the dummy events are sorted and picked up as a candidate to represent a dark event. The dummy event is called "a gluing passage dark event" since it injects a new passage (words) to glue existing word groups.

$$
C o_{i} \equiv \sum_{c_{j}} \max _{e_{k} \in c_{j}} \mathrm{Ja}\left(D E_{i}, e_{k}\right),
$$

The human's interpretation starts with the scenario map which the crystallization algorithm outputs. It includes "Interpret clusters", "Invent hypothetical scenarios", and "Infer the identity of dark events". The iteration in the annealing process is terminated when human converges into complete understanding, resulting in inventing many hypothetical scenarios. 


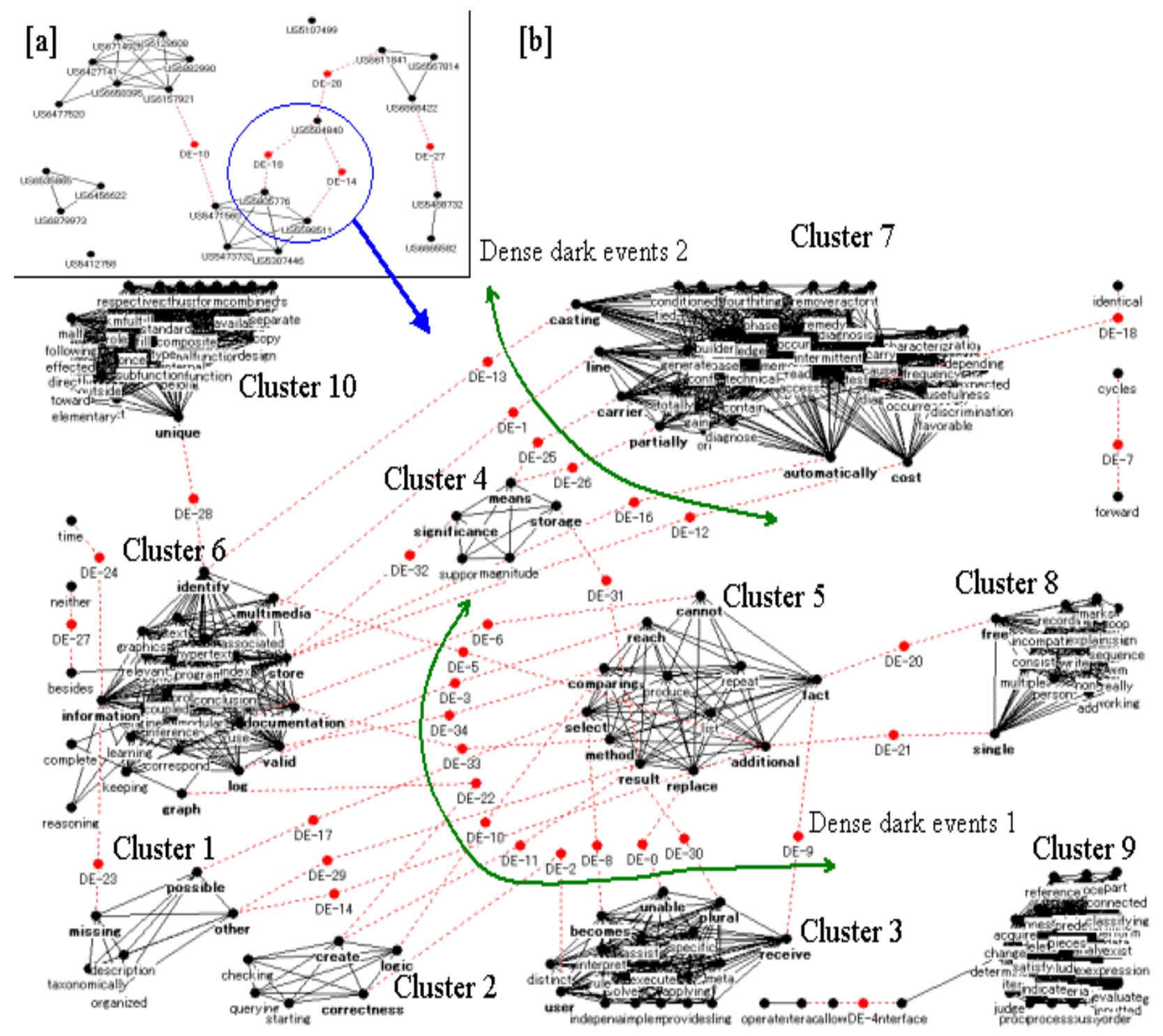

Fig.4 Scenario map derived from the human-interactive annealing; [a] for patents, and [b] for claims.

\section{Experiment}

The human-interactive annealing is applied to patent texts for acquiring opportunity in technology development. This section demonstrates an illustrative example for picking up promising technological elements by analyzing US patents on knowledge acquisition and discovery.

Twenty-three US patents are configured into baskets based on the US class identifiers. The US class represents tree-structured categories of technologies. The result is shown in fig. 3 (see [a]). Five dark events appear among five clusters on the scenario map. Here, we focus on the latent structure between the clusters 2 and 3. The dark events refer to the baskets, whose subjects are "US class identifier $=706 / 50$ : knowledge processing system $/$ having specific management of a knowledge", and "US class identifier $=706 / 59$ : knowledge processing system / creation or modification".

Then, we go on to the details of the patent texts. As an input data, we extracted claims in the patents 5805776 , and 5598511 in the cluster 2, and 5504840 in the cluster 3. Although the other portions of patent texts can be used, claims directly represents a means to solve a problem; technological elements of interest. They are processed with the human-interactive annealing, and visualized on a 
scenario map. The scenario map is shown in fig. 3 (see [b]).

Ten word clusters and thirty-three gluing passage dark events appear. A few isolated events also appear. The cluster 5 seems to play a role to connect the clusters 1,2 , $3,4,6$, and 8 . We focus on the area where the density of dark events is large; among the clusters $2,3,5$, and $6(=$ dense dark events 1 ), and among the clusters 4,6 , and 7 (= dense dark events 2). Before inventing scenario on technological elements in these areas, the meaning of the clusters must be interpreted. For example, the clusters 2, 3, 5 , and 6 are interpreted to represent ideas; "replace, compare, or select facts", "check or query logic", "interpret or execute rules", and "learn, infer, or identify". Table 1 summarizes the interpreted keywords for the relevant clusters.

TABLE 1

Interpretation of clusters.

\begin{tabular}{ll}
\hline \hline Cluster & Keywords from interpretation \\
\hline 2 & correct, check, logic, query \\
\hline 3 & interpret, execute, apply, implement, rule, user \\
\hline 4 & support, store, significance, magnitude \\
\hline 5 & replace, compare, select, produce, repeat, fact \\
\hline 6 & $\begin{array}{l}\text { learn, infer, identify, reason, text, document, } \\
\text { multimedia, graphics }\end{array}$ \\
\hline 7 & $\begin{array}{l}\text { diagnose, extract, frequency, occurrence, rate, } \\
\text { cost, knowledge base }\end{array}$ \\
\hline \hline
\end{tabular}

From the interpretation of the clusters, we invented a few scenarios incorporating the identity of gluing passage dark events in dense areas. Here we regard the dense area as a structure to represent a technical idea as a whole. Two examples are listed below. Although this analysis is for a simple demonstration purpose, it indicates how we should proceed to get an insight into a scenario for technology development.

- Enhance learning by replacing facts with the aid of interpretation of queries on expert knowledge (= dense dark events 1).

- Assign significance to knowledge inferred from multiple information (texts, documents, multimedia, graphics, etc. based on diagnosis by cross-validating knowledge (= dense dark events 2$)$.

\section{Conclusion}

Human-interactive annealing is a new method for understanding invisible but relevant events, and inventing hypothetical scenarios. The human-interactive annealing is elaborated for texts. It is applied to patents for acquiring opportunity in technology development. An illustrative experiment on discovering opportunity by analyzing US patent texts on knowledge acquisition is demonstrated. A few sample scenarios regarding emerging technological elements are obtained.

Popular approaches in technology development are engineering design methods such as TRIZ (Theory of Inventive Problem Solving in Russian), VE (Value Engineering), or Taguchi method. These methods mainly aim at utilizing precedent successful cases and optimizing combination of technological elements under cost and quality constraint. It gets, however, more important to identify invisible emerging technological elements to eliminate threat and to acquire opportunity in technology development. Instead of patent texts, we can also employ technical papers, and news and reports on technology. The human-interactive annealing is expected to shed new light on such problem domains.

\section{References}

[1] Y. Ohsawa eds., to be published, Chance discovery in real world decision making. Springer-Verlag, 2006.

[2] Y. Ohsawa, and P. McBurney eds., Chance discovery (Advanced information processing). Springer-Verlag, 2003.

[3] Y. Maeno, and Y. Ohsawa, "Human-computer interactive annealing for discovering invisible dark events," to appear, IEEE transactions on industrial electronics, 2006.

[4] Y. Maeno, and Y. Ohsawa, "Stable deterministic crystallization for discovering hidden hubs," to appear, IEEE International Conference on Systems, Man, and Cybernetics, Taipei, 2006.

[5] Y. Maeno, and Y. Ohsawa, "Crystallization Highlighting Hidden Leaders," Proceedings of IPMU (Information Processing and Management of Uncertainty in Knowledge-Based Systems) International Conference, Paris, 2006.

[6] K. Horie, Y. Maeno, and Y. Ohsawa, "Human-interactive annealing process with pictogram for extracting new scenarios for patent technology," to appear, CODATA (International Council for Science: Committee on Data for Science and Technology) International Conference, Beijing, 2006.

[7] M. Tory, and T. Moeller, "Human factors in visualization research," IEEE transactions on visualization and computer graphics, vol. 10, 2004, pp. 72-84.

[8] R. Kwok, J. Ma, and D. Zhou, "Improving group decision making: a fuzzy GSS approach," IEEE transactions on systems, man, and cybernetics Part C, vol. 32, 2002, pp. 54-63.

[9] R. Rush et. al, "Elicitation of knowledge from multiple experts using network Interface," IEEE transactions on knowledge and data engineering, vol. 9, 1997, pp. 688-696.

[10] T. Hastie, R. Tibshirani, and J. Friedman, The elements of statistical learning: Data mining, inference, and prediction (Springer series in statistics). Springer-Verlag, 2001. 